\title{
UDP Connectivity Performance in VoIP over WiMAX
}

\author{
Salma Mohmed Abdalh Mohamed ${ }^{1}$, Dr. Mohmmed Abakar ${ }^{2}$ \\ Department of Communication Engineering, Al-Neelain University
}

\begin{abstract}
In the recent years, WiMAX technology is widely used for wireless communication systems in many countries because it has rich set of features with promising broadband wireless access networks. The paper is introducing many cells with the Mobile WiMAX and Fixed WiMAX networks for better performance over the network protocol UDP. In paper, performance evaluation of WiMAX is done on software opnet. Various real life scenarios have been created to see how different factors such as distance, number of subscriber stations, different modulation schemes and packet size affect the performance of the WiMAX networks. Simulation of performance is carried out for UDP protocol traffic over fixed as well as mobile WiMAX. Throughput, average delay and average jitter are used as performance metrics in this study.
\end{abstract}

Keywords: WiMAX, UDP, Performance, throughput, QoS

\section{Introduction}

In the most recent decade, the telecommunication industry has advanced more quickly. Mobile telephony and the Internet have been widely embraced by populations around the globe. As the demand for mobile broadband is growing and evolving, this quick change in the communication way and the way we get information is continue to accelerate. WiMAX stands for Worldwide Interoperability for Microwave Access. WiMAX technology enables everpresent communication of wireless broadband service for fixed and/or mobile users. It opens doors to new players by offering new frequency allocations, global open standards, new networks and new business models. WiMAX networks are inherently simple, spectrally efficient and easy to deploy. Because WiMAX is a cost effective, standards based, wireless technology, it has already given rise to next generation applications, new chipsets and new devices. WiMAX is the other name of IEEE standard 802.16. It was a technology to offer the last-mile wireless broadband which is comparable with cable and DSL and where the cost is comparatively high. It's intended to deliver high speed data communication, and it also has the ability to maintain dedicated links and VoIP services at a reliable and high quality speed and allow the subscribers to connect to a wireless internet service provider without being at the office

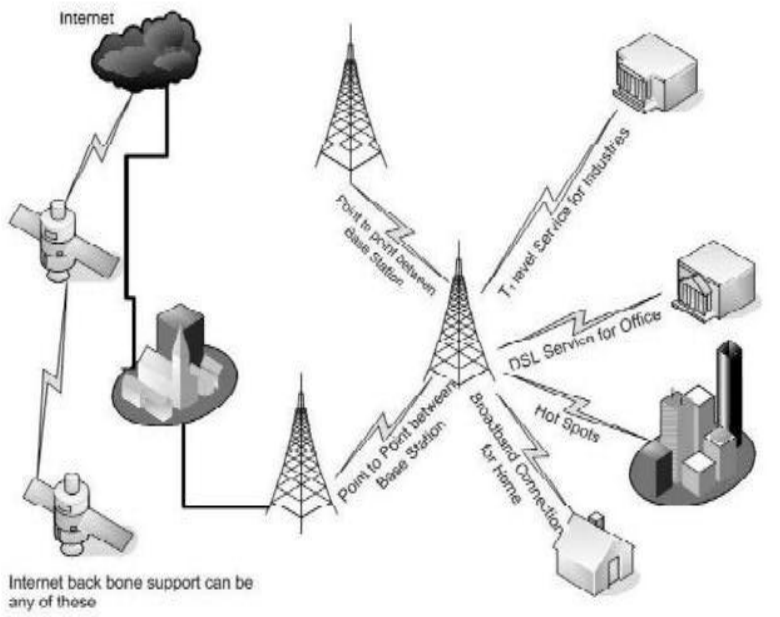

Figure 1 desk. WiMAX networks provides high data rates, last mile wireless access, point to multipoint communication, large frequency range and quality of services for various type of applications. Mobile WiMAX. fixed and mobile broadband is already a reality that presents tremendous opportunity to new industry entrants who are poised to capitalize on it.

There are two WiMAX Standards, IEEE 802.16d-2004 known as Fixed WiMAX and IEEE 802.16e-2005 known as

WiMAX handles the constantly increasing demands for broadband wireless applications. The bandwidth and range of WiMAX make it suitable for various potential applications. One of the main applications of the WiMAX is that it can be used in disaster recovery scenes where the wired networks have broken down. In recent many disasters, WiMAX networks were installed to help in recovery missions. Similarly, WiMAX also be used as a backup links where the traditional wired links breaks.

WiMAX mainly operates in two frequency ranges. One is high frequency, which ranges from $11-66 \mathrm{GHz}$ also known as licenced frequency band and another one is low frequency, $2 \mathrm{GHz}-11 \mathrm{GHz}$, referred to as unlicensed frequency band. While operating in high frequency range, Line of Sight (LOS) is essential. The other one, non- line of Sight is essential for the latter range.

The WiMAX Network technology is an evolutionary one as it uses orthogonal frequency division multiplexing which makes transmission resist fading and minimizes multipath effect. In addition, a WiMAX network can work as a pointto-point backhaul trunk with a transmission capability of 72 Mbps at a transmission distance over 30 miles. With its technological advantages of power, throughput, $3 \mathrm{G}$. It is the capability of WiMAX networks in providing high bandwidth with QoS deployed over large areas which is seen as key advantages of WIMAX infrastructure. 


\section{International Journal of Science and Research (IJSR) \\ ISSN (Online): 2319-7064}

Index Copernicus Value (2013): 6.14 | Impact Factor (2015): 6.391

\section{Divisions of WiMAX}

\section{A. Fixed WiMAX}

802.16d was developed specifically for the fixed wireless applications, because it does not support mobility, the terminal devices or Customer Premise Equipment are not constrained by battery operation or small form factor for handheld operation. This gives up both ends of the link to allow for some symmetry in performance between the CPE and the base station. Typically both the CPE and the base station can support high output power through the radio and antenna combined. The result is excellent over long distances.

Because of hardware and installation costs outdoor antennas are very expensive. The PHY layer in Wireless-MAN is basically based on OFDM. Its primary purpose is for access deployment where SSs are used as residential gateways deployed within home and business applications. OFDM supports sub channelization in the UL. Fixed WiMAX is very robust against multi-path propagation because it used an air interface based on orthogonal frequency division multiplexing (OFDM). It is based on IEEE 802.16 and will initially operate in the $2.3 \mathrm{GHz}, 2.5 \mathrm{GHz}$, and 3.4 to 3.8 $\mathrm{GHz}$ spectrum bands.

The IEEE 802.16-2004 standard is designed for fixed-access usage models. This standard may be referred to as "fixed wireless" because it uses a mounted antenna at the subscriber's site. The antenna is mounted to a roof or mast, similar to a satellite television dish. IEEE 802.16-2004 also addresses indoor installations, in which case it may not be as robust as in outdoor installations.

One of the essential components of WiMAX is OFDM. As stated in [2] Orthogonal Frequency Division Multiplexing (OFDM) breaks the wireless carrier into 256 sub-carriers.

\section{B. Mobile WiMAX}

The IEEE 802.16e standard is an amendment to the 802.162004 base specification and targets the mobile market by adding portability and the ability for mobile clients with IEEE 802.16e adapters to connect directly to the WiMAX network to the standard. The $802.16 \mathrm{e}$ standard uses Orthogonal Frequency Division Multiple Access (OFDMA), which is similar to OFDM in that it divides the carriers into multiple sub carriers. OFDMA, however, goes a step further by then grouping multiple subcarriers into sub-channels. A single client or subscriber station might transmit using all of the sub-channels within the carrier space, or multiple clients might transmit with each using a portion of the total number of sub-channels simultaneously. The Mobile WiMAX air interface adopts the Orthogonal Frequency Division Multiple Access (OFDMA) modulation scheme for improved multipath performance in non-line-of-sight environments. OFDMA assigns a subset of subcarriers to individual users and the transmission is simultaneous. Each OFDMA user transmits symbols using subcarriers that are orthogonal to other users. More than one subcarrier can be assigned to one user to support high data rates [3].

\section{WiMAX Architecture}

The WiMAX network reference model is unified network architecture for supporting fixed, roaming, and mobile deployments and is based on an IP service model. The overall network may be logically divided into three basic parts:

1) Mobile Stations (MS) used by the end user to access the network.

2) The access service network (ASN), which comprises one or more base stations and one or more ASN gateways that form the radio access network at the edge.

3) Connectivity service network (CSN), which provides IP connectivity and all the IP core network functions.

4) Some of the important functional entities are given as:

\section{A. Base Station (BS)}

The BS is responsible for providing the air interface to the MS. Additional functions that may be part of the BS are micro mobility management functions, such as handoff triggering and channel establishment, radio resource management, QoS policy enforcement, traffic classification, DHCP (Dynamic Host Control Protocol) proxy, key management, session management, and multicast group management.

\section{B. Access Service Network Gateway (ASN-GW )}

The ASN gateway typically acts as a layer 2 traffic aggregation point within an ASN. Additional functions that may be part of the ASN gateway include intra-ASN location management and paging, radio resource management and admission control, caching of subscriber profiles and encryption keys, authentication authorization, and accounting (AAA) client functionality, establishment and management of mobility tunnel with base stations, QoS and policy enforcement, foreign agent functionality for mobile IP, and routing to the certain CSN.

\section{Connectivity Service Network (CSN)}

The CSN provides connectivity to the Internet, Access Service network (ASN), other public networks, and corporate networks. The CSN is owned by the Network Service Provider and includes AAA servers that support authentication for the devices, users, and specific services. The CSN also provides per user policy management of QoS and security. The CSN is also responsible for IP address management, support for roaming between different NSPs, location management between ASNs, and mobility and roaming between ASNs.

\section{UDP ( User Datagram Protocol)}

The User Datagram Protocol (UDP) is a core member of the Internet protocol suite (the pattern of network protocols used for the Internet). With UDP, computer applications can send messages, or data, in this case known as datagram's, to another hosts in an Internet Protocol (IP) network without a communication to set up important transmission channels or data paths.

As this is normally Internet protocol over unreliable media, there is no surety of delivery, ordering, or any protection. UDP provides checksum for data integrity, and port 


\section{International Journal of Science and Research (IJSR) \\ ISSN (Online): 2319-7064}

Index Copernicus Value (2013): 6.14 | Impact Factor (2015): 6.391

numbers for addressing different functions at the source and destination of the datagram [14]. UDP is suitable for the purpose where error checking and correction is not necessary or done in the application, neglecting the overhead of such processing at the network interface level. Time-sensitive applications also use UDP because dropping packets is referred to waiting for delayed packets, which may not be a way in a real-time system. If error correction techniques are needed at the network interface level, another application may use the Transmission Control Protocol (TCP) or Stream Control Transmission Protocol (SCTP) which are designed for this purpose.

\section{Proposed Scheme}

\section{A. Proposed Method}

Department of defense war fighting concepts leverage information superiority and require vast improvements in information transfer in terms of higher bandwidth, Quality of Service (QoS) support and connection to a high speed backbone. The new IEEE 802.16 broadband wireless access system is a viable alternative that can meet such requirements. In addition, this network can be swiftly deployed to interconnect the military theater, emergency response, and disaster relief operations to the backbone. Due to the diverse multimedia traffic with different priorities and QoS requirements, it is a well-known fact that it is imperative to provide QoS support in military networks. However, the IEEE 802.16 provides only signaling mechanisms, but does not specify any scheduling or admission control algorithms that ultimately provide QoS support. In this paper we introduce a new scheduling algorithm for IEEE 802.16 broadband wireless access standard. The proposed solution which is practical and compatible to the IEEE 802.16 standard provides QoS support to different traffic classes. To the best of our knowledge this is the first such algorithm. The simulation studies show that the proposed solution includes QoS support for all types of traffic classes as defined by the standard. We have shown the relationship between traffic characteristics and its QoS requirements and the network performance. This study will help network architects to decide the system parameters as well as the kind of traffic characteristics for which the network can provide QoS support.

\section{B. Methodology}

1) Consider the input parameters such as sample frequency, max memory and sample period.

2) Defining a QPSK modulation for the generation WIMAX

3) Formation of WIMAX framing structure contains the data transmission from transmitter and receiver using IFFT in transmitter and FFT in receiver.

4) Adding the even and odd frame guard in the formation of cyclic prefix here we are implementing 14 carriers in to their pre sub cluster mapping.

5) In the receiving part we are calculating the sampling rates for which we are applying the correctness of resampling.

6) After the formation of WIMAX network we are applying this to UDP traffic.
7) In UDP traffic we check the average throughput for different modulation and encoding schemes. The proposed methodology is needed to be implemented in a tool. The tool opted for simulation of the proposed work is Opnet.

\section{Results and Discussion}

Simulation scenarios have been considered for evaluation of WiMAX network. The effect of following parameters has been analyzed:

1) Number of Subscriber Station

2) Distance between Base station(BS) and SS

Different Modulation schemes

\section{A. Impact of Service Stations (SS)}

Fig. 2 shows average throughput as a function of number of nodes. It is observed that the throughput steadily increases as the number of nodes is increased. The reason is that as the number of nodes is increased, the Number of packets being transmitted also increases. These include data packets as well as control packets that are exchanged between the SS and BS. So for two nodes, the throughput is around 486 kbps. For 20 nodes, the value reaches around $4715 \mathrm{kbps}$ for mobile WIMAX. Fixed WiMAX also gives almost similar values.

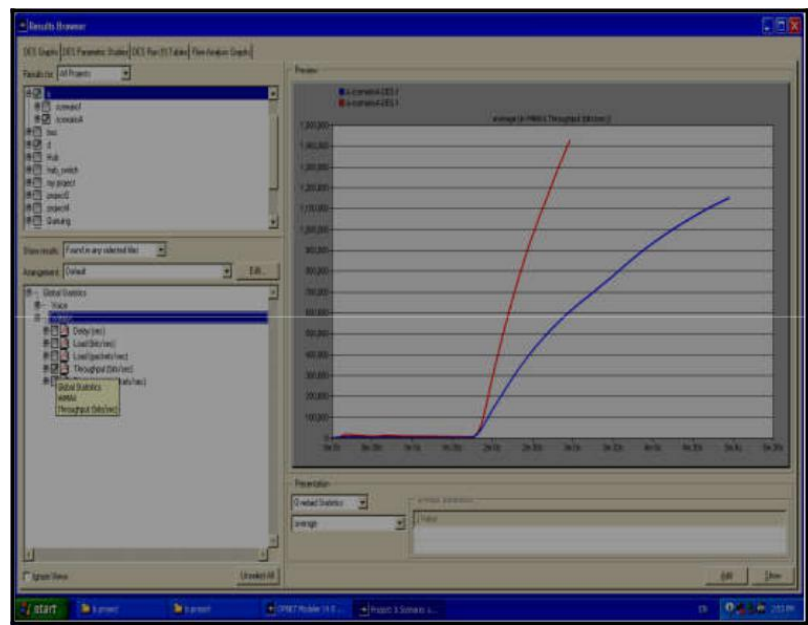

Figure 2: Average throughput (kbps) as a function of number of nodes

Fig. 3 shows average delay as a function of the number of subscriber stations in vicinity of base station. It is observed that with an increase in the number of subscriber stations, average delay initially increases and then becomes almost constant. Also, the average delays in case of $802.16 \mathrm{e}$ are smaller as compared to $802.16 \mathrm{~d}$. This is due to use of Scalable OFDMA in case of $802.16 \mathrm{e}$ as opposed to $802.16 \mathrm{~d}$ where classical OFDM is used. 


\section{International Journal of Science and Research (IJSR) \\ ISSN (Online): 2319-7064}

Index Copernicus Value (2013): 6.14 | Impact Factor (2015): 6.391

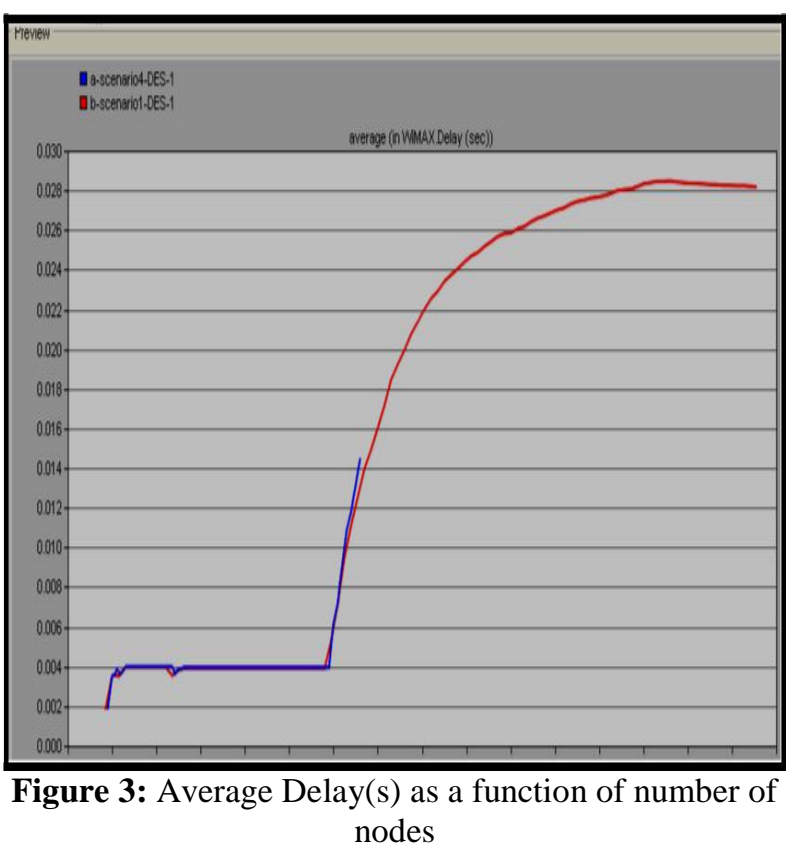

Fig. 4 shows average jitter as a function of the number of nodes. For mobile WIMAX, average jitter is remaining constant and negligible for all practical purposes. For fixed WiMAX, there is increase in delay up to 6 nodes and then it becomes constant.

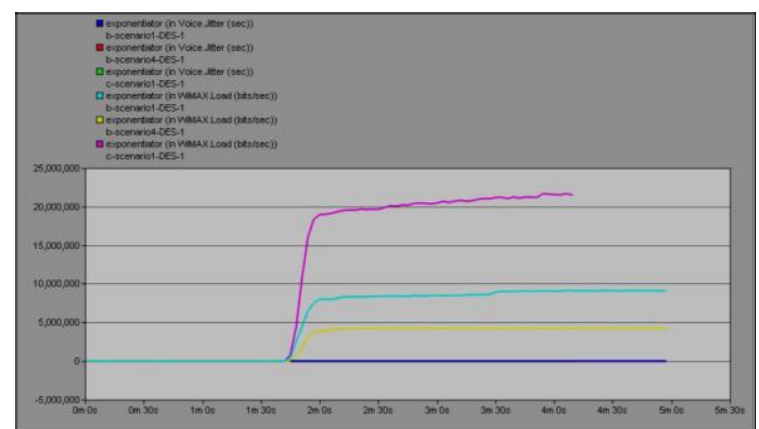

Figure 4: Average jitter(s) as a function of number of nodes

\section{B. Impact of distance between BS and SS}

Fig. 5 shows average throughput as a function of distance between base station and subscriber stations. It has been observed that with increase in distance the throughput of both $802.16 \mathrm{~d}$ and $802.16 \mathrm{e}$ remains almost constant, however, beyond a Distance of $8 \mathrm{~km}$, the throughput of 802.16e drops drastically.

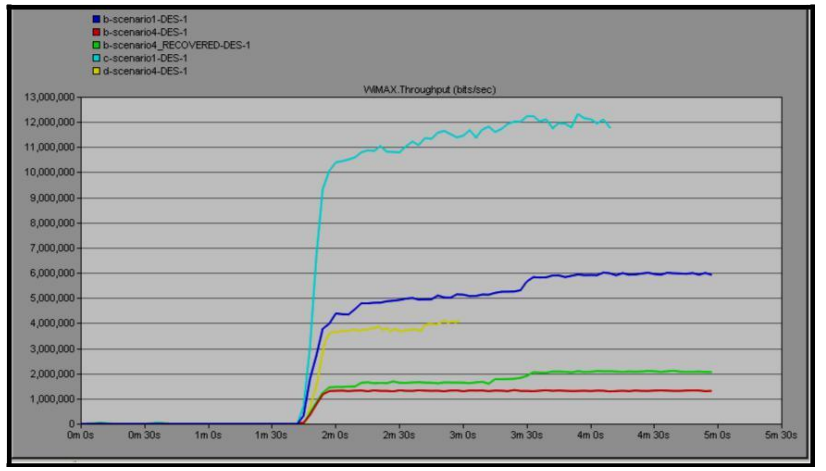

Figure 5: Average throughput (kbps) as a function of distance between BS and SS
Fig. 6 shows the average delay as a function of distance. Average delay remains almost constant as distance is varied for both fixed and mobile WIMAX. Mobile WIMAX has lesser delay than fixed WIMAX. The average jitters as function of distance. The average jitter does not show significant variation with distance for both fixed and mobile WiMAX. Again mobile WiMAX performs better than fixed WiMAX. The values are very low and practically insignificant.

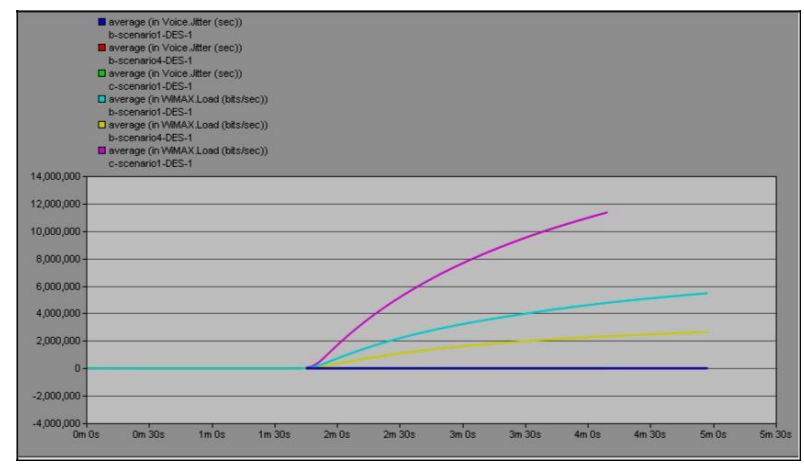

Figure 6: Average jitter(s) as a function of distance between BS and SS

\section{Conclusions}

In this paper, the performance study for mobile WiMAX, fixed WiMAX has been presented using Opnet. Result obtained from simulation shows how several performance metrics such as throughput, delay are affected by change in factors like number of nodes, modulation scheme, distance between BS and SS. It is also found that fixed WiMAX can support up to larger distance in comparison to mobile WiMAX. Other factors that do affect the performance of WiMAX network are antenna gain, MIMO gain, and output power of $\mathrm{BS}$, TDD ratio, $\mathrm{CPE}$ antenna gain and receiver antenna gain of BS. Therefore, by choosing appropriate values of different factors/parameters according to channel condition and other available resources, performance of WiMAX can be optimized.

\section{References}

[1] A.F.M Sultanul Kabir1,Md.Razib Hayat Khan2,Abul Ahsan Md.Mahmudul Haque1 and Mohammad Saiful Islam Mamun, "WiMAX or Wi-Fi"2003

[2] Ali Nawaz Naqvi, Ash Mohammad Abbas, Tofik Ali Chouhan, "Performance Evaluation of Fixed and Mobile WiMAX Networks for UDP Traffic",, October 2012

[3] Mike Linsey, Prosy Nalweyiso, Preethi Srinivasan, Lou Ann Stroup, Narasimhan Vasudevan, "Mobile WiMAX”, April 2010

[4] Claudio Cicconetti Alessandro Erta, Luciano Lenzini, and Enzo Mingozzi "Performance Evaluation of IEEE 802.16 MAC for QoS Support”, January 2007

[5] Tarek Bchini, Nabil Tabbane, Tunisia T.Bchini, Emmanuel Chaput "QoS in IEEE 802.16e for VoIP and Video with mechanism of Soft Handover - FBSS in Highway, 2009.

[6] Chin "Performance Analysis of Broadband Wireless Access Systems with Antenna Selection" 2006

Volume 5 Issue 6, June 2016 www.ijsr.net 


\section{International Journal of Science and Research (IJSR) \\ ISSN (Online): 2319-7064}

Index Copernicus Value (2013): 6.14 | Impact Factor (2015): 6.391

[7] Omar Arafat, K. Dimyati, "5 Performance Parameter of Mobile WiMAX : A Study on the Physical Layer of Mobile WiMAX under Different Communication Channels \& Modulation Technique" 2010 Applications.

[8] S. Askar, H.S AI-Raweshidy "Performance Evaluation Of IEEE 802.16-2004.

[9] IEEE DRAFT Standard for Local and metropolitan area networks Part 16: Air Interface for Broadband Wireless Access Systems (Revision of IEEE Std 802.16-2004).

[10] Wei Wang Sharif, Hempel, M. ; Ting Zhou "Implementation and performance evaluation of QoS scheduling algorithms in Mobile WiMAX ns-2 simulator.2004

[11] Safak, A. Ozdem, M.S., Uyanik, E. Signal Processing and Communications Applications, 2007.

[12] Wang, H. Hamza, M. Hempel and Ting Zhou, "Implementation and Performance Evaluation of QoS Scheduling Algorithms in Mobile WiMAX NS-2 Simulator, 1-6, 2010.

[13] Najma, S. Karim and M. Ahmed, "Performance Study on Optimal Boundary per WiMAX Cell", Artech House, 2000.

[14]Zhibin Zeng; Dian Xie; Yaqian Huang, March 2012.

[15] Stefania Sesia, Issam Toufik, Matthew Baker, The UMTS Long Term Evolution,2011

[16] S. B. Slimane, 3, 2000.

[17] R. W. Bauml, R. F. H. Fischer, and J. B. Huber,Oct. 1996.

[18] Tao Jiang, " An Overview: Peak -to-Average Power Ratio Reduction Techniques for OFDM signals",June 2008.

[19] X.Li and L.J.Cimini., (1998). "Effect of clipping and filtering on the performance of OFDM". IEEE Communications Letters, (May 1998), X.Huang, J.Lu, J.Zheng, and J.Chuang.,(2001).

[20] "Reduction of peak-to-average power ratio of OFDM signals with companding transform", (April 2001).

[21] M.Sharif, M.Gharayi-Alkhansari, and B.H.Khalaj., (2003).

[22] Improvement in OFDM System using SLM with Adaptive Nonlinear Estimator", 2009.

[23] Guangyue Lu, Ping Wu and Catharina CarlemalmLogothetis," PAPR reduction for real baseband OFDM signals", 2008.

[24] Wu and W. Y. Zou, "Orthogonal frequency division multiplexing, pp. 392-399, Aug. 1995

Volume 5 Issue 6, June 2016 www.ijsr.net 